\title{
Optical Spectroscopic Properties Recorded for Simple BOPHY Dyes in Condensed Media: The Mirror-Symmetry Factor
}

\author{
Owen J. Woodford, ${ }^{a}$ Raymond Ziessel, ${ }^{a}$ Anthony Harriman, ${ }^{\mathrm{a},{ }^{*}}$ Corinne Wills, ${ }^{\mathrm{b}}$ Abdulrahman A. \\ Alsimaree $^{c}$ and Julian G. Knight ${ }^{c}$
}

(a) Molecular Photonics Laboratory, School of Natural and Environmental Sciences, Bedson Building, Newcastle University, Newcastle upon Tyne, NE1 7RU, United Kingdom. (b) NMR Laboratory, School of Natural and Environmental Sciences, Bedson Building, Newcastle University, Newcastle upon Tyne, NE1 7RU, United Kingdom. (c) School of Natural and Environmental Sciences, Bedson Building, Newcastle University, Newcastle upon Tyne, NE1 7RU, United Kingdom.

Corresponding author: Anthony.harriman@ncl.ac.uk

\begin{abstract}
The BOPHY structural scaffold provides opportunities for the synthesis of innumerable derivatives with linear geometries and well-controlled $\pi$-conjugation pathways. The simpler BOPHY chromophores are highly fluorescent but exhibit poor mirror symmetry between absorption and fluorescence spectra at ambient temperature. In particular, the absorption (and excitation) spectra are broad and appear as two overlapping band of comparable intensity. In constrained media, such as low-temperature rigid glasses or stretched poly(ethylene) films, mirror symmetry is restored. Analysis of the temperature dependence recorded for simple BOPHY derivatives indicates that the vibronic envelope accompanying the electronic transitions can be well described in terms of lowand medium-frequency modes. Whereas the fluorescence spectral profile is only weakly dependent on temperature, the excitation spectrum is far more affected. The magnitude of the low-frequency mode, and the associated electron-phonon coupling, increase substantially with increasing temperature and is responsible for temperature broadening and distortion of the excitation spectrum in solution. This critical low-frequency vibronic mode is associated with out-of-plane torsional bending of the BOPHY unit. Variable temperature NMR studies failed to provide unequivocal evidence for conformational changes of one of the derivatives over the temperature range 193-353K.
\end{abstract}

Key words: Fluorescence : Photophysics : BODIPY dyes : Huang-Rhys factor : Temperature effects :

\section{Introduction}

We have an almost insatiable appetite for highly fluorescent dyes and numerous such families are known, each possessing virtues and weaknesses. One particular family, namely the boron dipyrromethene (BODIPY) collection of dyes [1-4], has been especially prevalent in recent years and has found many practical applications in biology, medicine, analytical chemistry and materials science. From the BODIPY stable has emerged a new class of robust, fluorescent dyes of the bis(difluoroboryl)1,2-bis((1H-pyrrol-2-yl)methylene)hydrazine family [5,6], known generically as BOPHY derivatives. These compounds have yet to be fully developed but appear to be attractive in terms of building linear arrays by accretion of several complementary BOPHY-based modules into a strand. An important issue relating to the BOPHY family concerns the relatively high-energy absorption and emission transitions, which occur around 440-460 $\mathrm{nm}$ for the unsubstituted derivatives [5]. Although these chromophores are relatively new, the basic conjugation pathway has been extended by both incorporation of styryl units [7] and fusing aromatic residues to the central platform [8]. This gives access to BOPHY derivatives absorbing in the far-red region. Theoretical studies have described [9] how various substituents located at different sites on the BOPHY core interact to push the absorption transitions towards lower energy. Intersystem-crossing to the corresponding triplet-excited state, which is 
ineffective for the parent compound, can be promoted by incorporation of iodine atoms in the central BOPHY core [10]. Secondary chromophores, most notably perylene, can be attached to the BOPHY unit so as to increase the light-harvesting capability [6]. In summary, the field is highly active and progressive.

The parent BOPHY module is strongly fluorescent but has been reported [5] to lack mirror symmetry between absorption and fluorescence transitions, at least in fluid solution. In fact, the vibronic envelopes for the lowest-energy absorption and emission bands appear to be quite disparate despite referring to a single optical transition. Driven by the usefulness of these BOPHY fluorophores, we have undertaken an investigation into the environmental factors influencing the optical properties of the parent BOPHY and the corresponding tetra-methylated derivative. It will be shown that the simple spectral patterns observed in a low-temperature glass become much more complex in fluid solution at ambient temperature. Transient absorption studies carried out in fluid solution have been interpreted [11] in terms of fast vibrational cooling, slow electronic relaxation and an intermediate process occurring on some tens of picoseconds. This latter relaxation was assigned to a possible conformational exchange but without involvement of a separate electronic state. We, and others, had previously shown that internal conversion in conventional BODIPY dyes is extremely fast $[12,13]$ but does not involve any unusual conformational change. Theoretical calculations [9] made for BOPHY indicate that the $\mathrm{BF}_{2}$ units are out-of-plane with the fused 5- and 6-membered rings, with the lowestenergy structure having these $\mathrm{BF}_{2}$ groups on opposite planes of the aryl core. Various calculations $[5,9,14]$ have suggested the $\mathrm{N}-\mathrm{N}$ vibration might have special significance in determining the optical band shape. All these studies argue the case for a single optical transition being responsible for the absorption band appearing in the blue-violet region. Our spectroscopic studies are aimed at seeking experimental support for the conclusions raised by the quantum chemical calculations and further clarifying the cause of the restricted mirror symmetry observed in fluid solution.
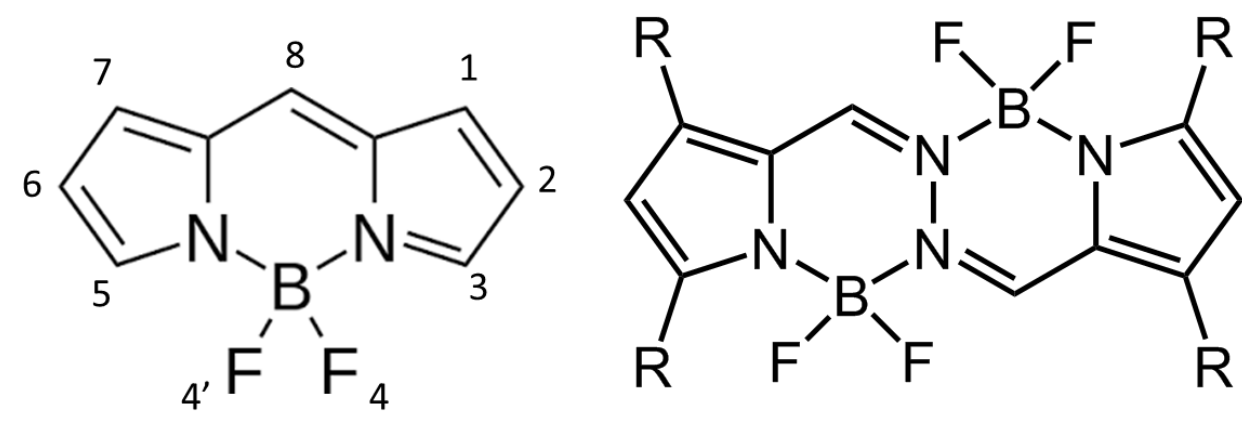

$\mathrm{R}=\mathrm{H}$ or $\mathrm{CH}_{3}$

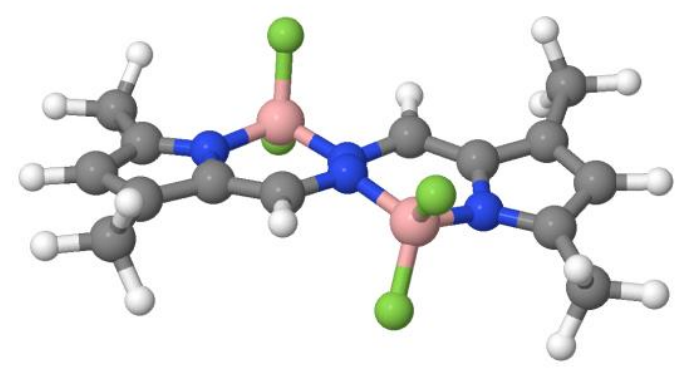

Figure 1. Chemical formulae for the popular BODIPY chromophore (left-hand side) and for the BOPHY chromophore (right-hand side). Note, BOPHY $\mathrm{R}=\mathrm{H}, \mathrm{TM}-\mathrm{BOPHY} \mathrm{R}=\mathrm{CH}_{3}$. Also shown is a computed structure (DFT/PBE0/6-311(d,s)/PCM/CHCl $)$ for TM-BOPHY in a solvent reservoir illustrating the nonplanar geometry. 


\section{Results and Discussion}

\section{Spectroscopic properties in fluid solution}

In this study we report optical spectroscopic information for two simple BOPHY derivatives. Both compounds, abbreviated as BOPHY and TM-BOPHY (Figure 1), have been reported previously in the literature [5]. Samples were synthesized and characterised as before. Small samples were further purified by preparative TLC immediately before use. The discussion focusses on TM-BOPHY while most of the corresponding studies made with BOPHY are relegated to the Supporting Information to avoid too much duplication. Any significant differences between the two sets of data are highlighted. To a large degree, our results collected for the chromophores in fluid solution at ambient temperature are comparable to literature reports.

The absorption spectra of BOPHY (Figure S1) and TM-BOPHY (Figure 1) recorded in 2methyltetrahydrofuran (MTHF) exhibit the two closely-spaced peaks reported previously in the literature. For TM-BOPHY, the absorption spectral profile is red-shifted by ca. $20 \mathrm{~nm}$ compared to the unadorned chromophore. The fluorescence spectrum for TM-BOPHY in MTHF displays a typical vibronic progression (Figure 2) with a maximum at $490 \mathrm{~nm}$. This corresponds to a Stokes' shift of 1,000 $\mathrm{cm}^{-1}$. The excitation spectrum gives a reasonable, but not perfect, match to the absorption spectrum (Figure 2); the match is improved for BOPHY (Figure S1). It was observed that TM-BOPHY followed the Beer-Lambert law over a concentration range from $300 \mu \mathrm{M}$ to $0.5 \mu \mathrm{M}$ and no sign of self-aggregation could be detected. Indeed, the absorption spectral profile remained strictly independent of solute concentration in MTHF.

Table 1. Compilation of the photophysical properties derived for the two compounds in 2methyltetrahydrofuran at room temperature.

\begin{tabular}{|l|l|c|c|c|c|c|c|}
\hline \multicolumn{1}{|c|}{ Cmpd } & $\varepsilon / \mathbf{M}^{-1} \mathbf{c m}^{-1}$ & $\mathbf{f}$ & $\Phi_{\mathrm{F}}$ & $\tau_{\mathrm{s}} / \mathbf{n s}$ & $\mathbf{k}_{\mathrm{RAD}} / \mathbf{s}^{-1}$ & $\mathbf{k}_{\mathrm{SB}} / \mathbf{s}^{-1}$ & $\mathbf{k}_{\mathrm{NR}} / \mathbf{s}^{-1}$ \\
\hline BOPHY & 38,500 & 0.60 & 0.65 & 2.4 & $2.7 \times 10^{8}$ & $2.8 \times 10^{8}$ & $1.5 \times 10^{8}$ \\
\hline TM-BOPHY & 37,500 & 0.56 & 0.75 & 2.7 & $2.8 \times 10^{8}$ & $2.9 \times 10^{8}$ & $8.9 \times 10^{7}$ \\
\hline
\end{tabular}

The molar absorption coefficient was found to be around $40,000 \mathrm{M}^{-1} \mathrm{~cm}^{-1}$ in dichloromethane solution, for both chromophores, in agreement with early reports $[5,6]$. These values were used to calculate the oscillator strength ( $f$ ) for the absorption profile by integration over the region 370-500 nm. In our hands, the fluorescence quantum yield $\left(\Phi_{\mathrm{F}}\right)$ for TM-BOPHY in $\mathrm{CH}_{2} \mathrm{Cl}_{2}$, was found to be 0.75 ; that for BOPHY was somewhat lower at 0.65 . Under these conditions, the time-resolved fluorescence decay profiles were well represented as single-exponential processes. A compilation of the important photophysical properties for the two compounds is given in Table 1 . The radiative rate constant $\left(k_{\text {RAD }}\right.$ $\left.=\Phi_{\mathrm{F}} / \tau_{\mathrm{S}}\right)$ and excited-singlet state lifetime $\left(\tau_{\mathrm{S}}\right)$ do not change significantly with addition of the methyl groups while the radiative rate constant $\left(\mathrm{k}_{\mathrm{SB}}\right)$ calculated from the Strickler-Berg expression [15] is in quite good agreement with the experimental value. This is surprising because a general condition for using the Strickler-Berg expression is that good mirror-symmetry exists. This is clearly not the case with BOPHY and TM-BOPHY and we return to this point later. Also given in Table 1 are the corresponding rate constants for radiationless decay $\left(\mathrm{k}_{\mathrm{NR}}\right)$ of the first-excited singlet state. These values are much higher than found for related BODIPY fluorophores and remain competitive with those for radiative decay. It should also be mentioned that our derived $\Phi_{\mathrm{F}}$ values are notably lower than values reported in the literature, where $\Phi_{\mathrm{F}}$ values approaching unity can be found $[5-7,8 \mathrm{~d}]$. 


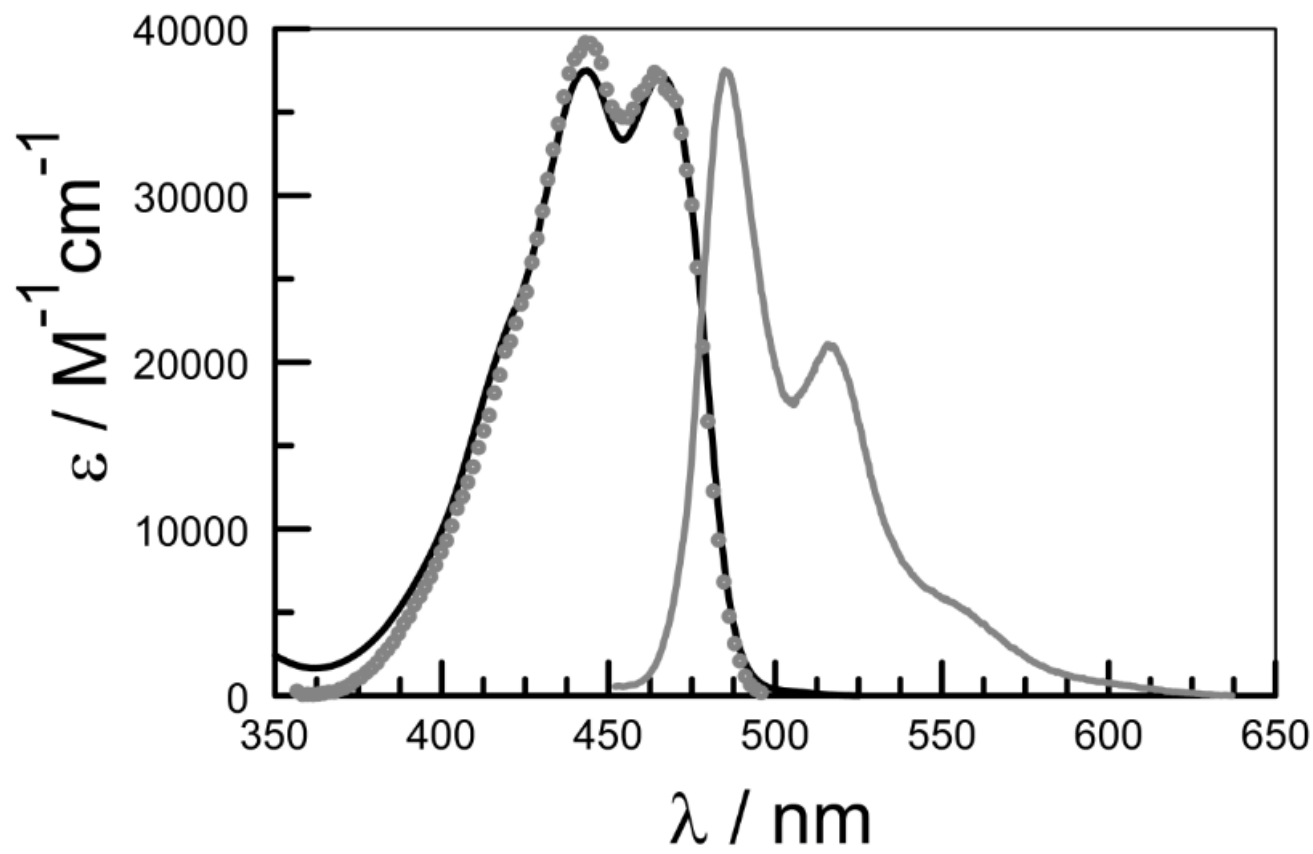

Figure 2. Normalised absorption (black curve), fluorescence (grey curve) and excitation (grey points) spectra recorded for TM-BOPHY in 2-methyltetrahydrofuran at room temperature. The excitation wavelength was $415 \mathrm{~nm}$ while the emission wavelength used for the excitation spectrum was $600 \mathrm{~nm}$.

Table 2. Effect of solvent on selected photophysical properties recorded for TM-BOPHY at room temperature.

\begin{tabular}{|c|c|c|c|c|c|}
\hline Solvent & $P_{\text {SOL }}{ }^{(e)}$ & $\lambda_{\mathrm{ABS}} / \mathrm{nm}$ & $\lambda_{\mathrm{FLU}} / \mathrm{nm}$ & $\Phi_{\mathrm{F}}$ & $\Delta_{\mathrm{ss}} / \mathrm{cm}^{-1}$ \\
\hline $\mathrm{C}_{6} \mathrm{H}_{12}$ & 11.49 & 470 & 485 & 0.78 & 660 \\
\hline $\mathrm{CH}_{2} \mathrm{Cl}_{2}$ & 6.49 & 468 & 487 & 0.75 & 830 \\
\hline MTHF & 9.95 & 466 & 485 & 0.74 & 840 \\
\hline Acetone & 6.46 & 461 & 483 & 0.71 & 990 \\
\hline $\mathrm{CH}_{3} \mathrm{CN}$ & 4.91 & 459 & 482 & 0.65 & 1,040 \\
\hline $\mathrm{DMF}^{\text {(a) }}$ & 7.68 & 463 & 486 & 0.72 & 1,020 \\
\hline $\mathrm{CH}_{3} \mathrm{OH}$ & 3.08 & 461 & 482 & 0.71 & 950 \\
\hline Benzonitrile & 12.85 & 470 & 492 & 0.74 & 950 \\
\hline $\mathrm{BuCN}^{(\mathrm{b})}$ & 8.64 & 463 & 484 & 0.69 & 940 \\
\hline DMSO $^{(c)}$ & 7.94 & 464 & 488 & 0.67 & 1,060 \\
\hline PDMS $^{\text {(d) }}$ & NA & 467 & 484 & 0.78 & 750 \\
\hline
\end{tabular}

(a) N,N-Dimethylformamide. (b) Butyronitrile. (c) Dimethylsulfoxide. (d) Polydimethylsiloxane. (e) Solvent polarizability function taken from Ref. 00.

Transient absorption spectroscopy was used to further probe the photophysics of TM-BOPHY in solution but no meta-stable species were observed. In deaerated solution, laser excitation did not result in detectable levels of a long-lived, excited-triplet state. Addition of iodomethane $(20 \% \mathrm{v} / \mathrm{v})$ to BOPHY in MTHF at 77K resulted in the appearance of phosphorescence (Figure S2), but no such emission was seen in the absence of the spin-orbit promotor. The emission maximum for the phosphorescence is found at $600 \mathrm{~nm}$ and $630 \mathrm{~nm}$ for BOPHY and TM-BOPHY, respectively. This corresponds to a singlet-triplet energy gap of some $5,000 \mathrm{~cm}^{-1}$. The vibronic progression for the phosphorescence spectrum is similar to that of the fluorescence so little change in the molecular 
geometry occurs on accessing the triplet-state. This puts the triplet state at a relatively high energy compared to that seen for simple BODIPY dyes.

The fluorescence quantum yield was measured for TM-BOPHY in a range of organic solvents but did not show a marked sensitivity towards the nature of the solvent. The absorption and fluorescence maxima vary slightly throughout this range of solvents, as does the Stokes' shift. These maxima can be compared with the solvent polarizability function $\left(\mathrm{P}_{\mathrm{sol}}\right)$ calculated [16] from the composition of the solvent (Table 2). The result is a surprisingly linear correlation for both absorption and fluorescence maxima, with comparable gradients being observed (Figure 3). Similar results were found for BOPHY. In the main, the optical processes are determined by dispersion interactions between the chromophore and the surrounding solvent [17]. It is interesting, in view of the poor mirror symmetry, that both absorption and emission spectra are affected to similar degrees. It appears that the unadorned BOPHY chromophore is not solvato-chromic and that there are no indications for the involvement of charge-transfer interactions.

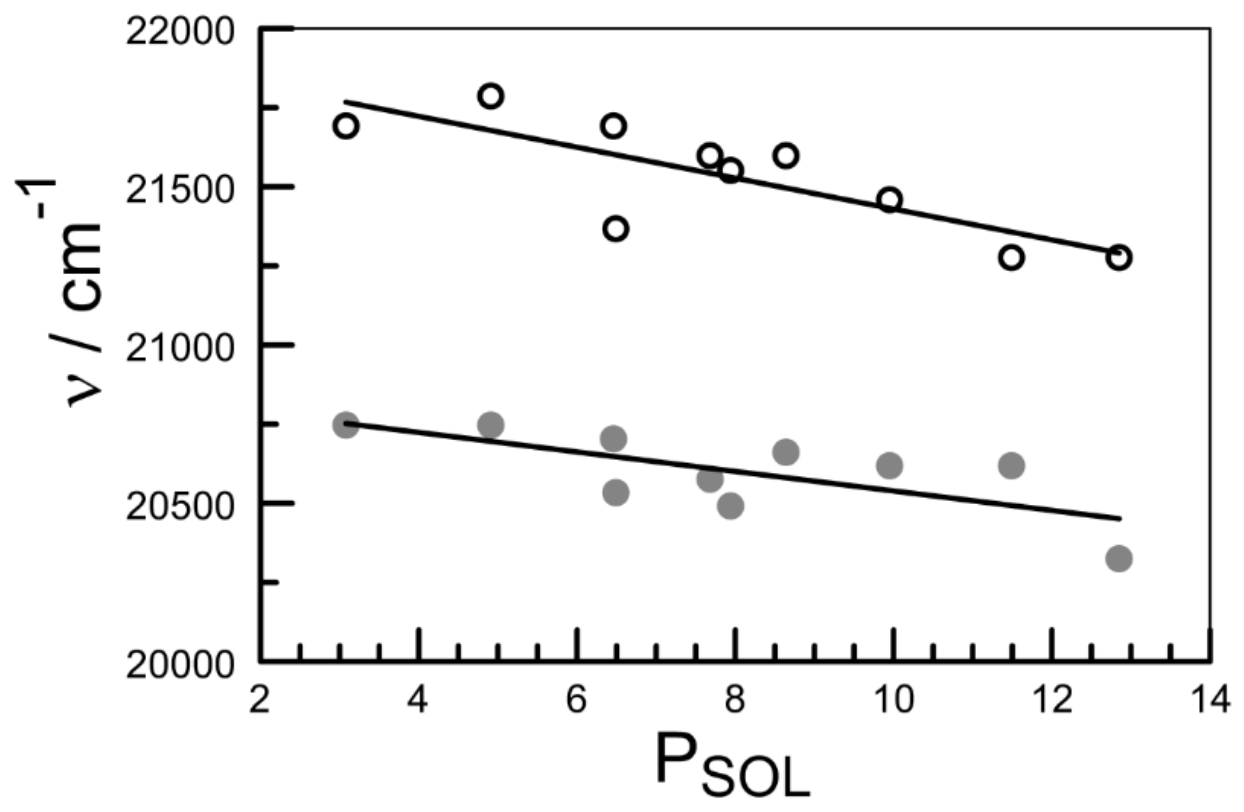

Figure 3. Effect of changes in solvent polarizability on the absorption (open circles) and fluorescence (grey points) maxima as determined for TM-BOPHY in various solvents at room temperature.

\section{Spectroscopic properties in constrained environments}

Both BOPHY derivatives show poor mirror symmetry in fluid solution at room temperature. The problem is easily traced to the absorption spectral profile, which is much broader than the emission spectrum and has the appearance of two overlapping transitions of comparable energy. To further examine this situation, we turn to low-temperature emission spectroscopy. Firstly, TM-BOPHY was studied in MTHF at $80 \mathrm{~K}$ after cooling very slowly from room temperature (Figure S3). The solvent freezes at 137K but does not form a rigid glass until around 100K [18]. Now, the fluorescence spectral profile recorded in the rigid glass is much better resolved than seen in fluid solution and is subjected to a modest blue shift. Apart from the band narrowing, vitrification of the solvent has little general effect. In marked contrast, the excitation spectra recorded in fluid solution and the rigid glass are very different (Figure S4). At $80 \mathrm{~K}$, the excitation spectrum is well resolved and slightly red shifted. Mirror 
symmetry between fluorescence and excitation spectra is now quite good. Thus, moving to a rigid matrix at low temperature has reduced the excitation spectrum to what appears to be a single vibronic progression. The effect is nicely replicated for BOPHY (Figure 4).

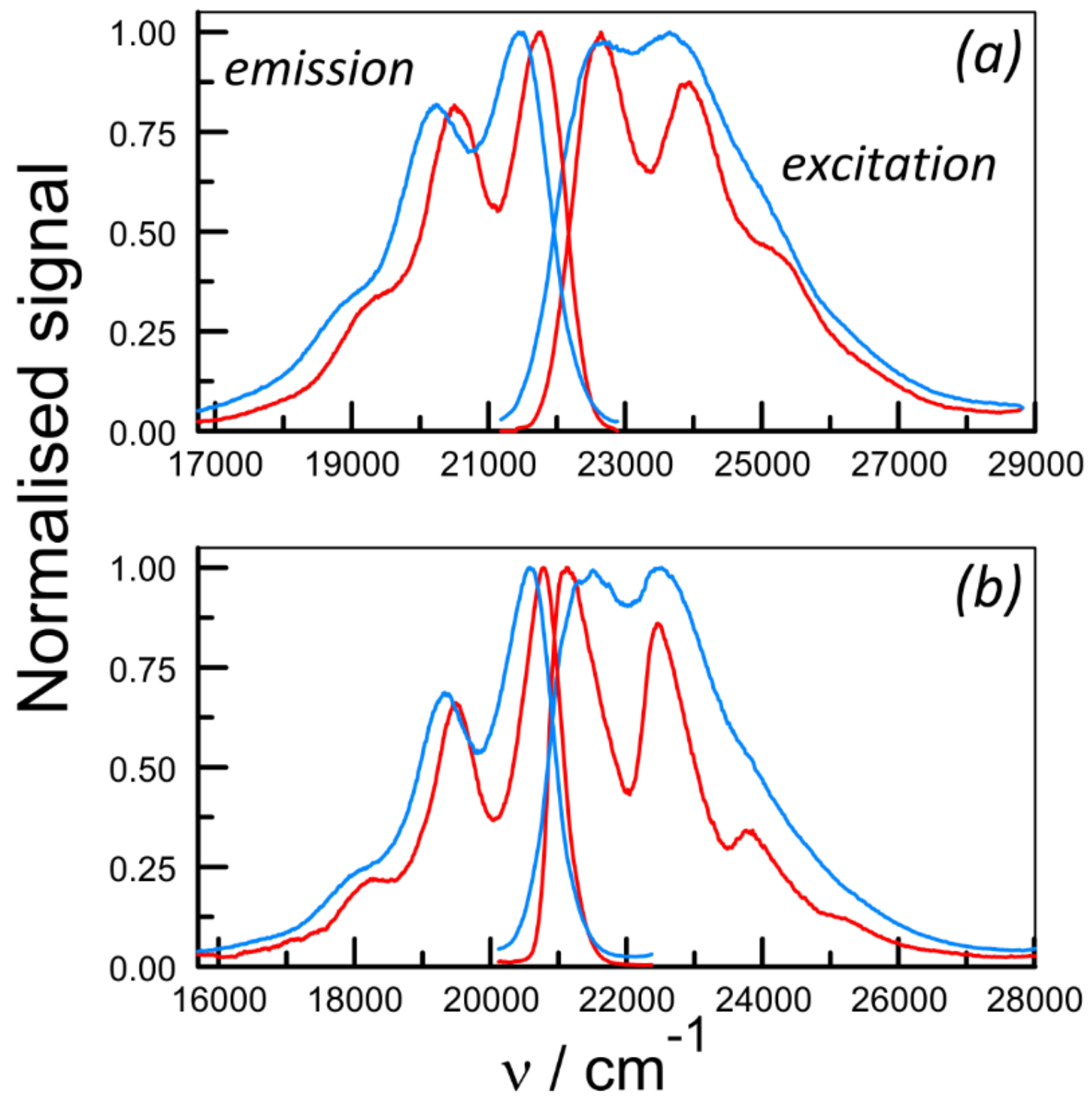

Figure 4. Reduced excitation and fluorescence spectra recorded for BOPHY (upper panel) and TMBOPHY (lower panel) in MTHF at 80K (red curves) and room temperature (blue curves) under otherwise identical conditions. The excitation wavelength used for fluorescence measurements was $425 \mathrm{~nm}$ while excitation spectra were recorded for fluorescence at $560 \mathrm{~nm}$.

A common strategy used to measure fluorescence polarisation is to orientate the fluorophore along a single axis imposed by a stretched polyethylene film [19]. Indirectly, this permits restricting the orientational dynamics of the chromophore without the need to use low temperatures. Quite remarkably, it was observed that TM-BOPHY aligned in stretched polyethylene film displays an excitation spectrum comparable to that seen in a rigid glass at low temperature (Figure S5). It would seem that, in restricting the molecular orientation by the polymer groove, access to the situation that causes the unusual excitation spectrum is denied. This effect cannot be reproduced by dispersing TMBOPHY in other matrices. For example, the fluorophore was immobilised in a polythene melt, in a 
Zeonex 480 cast film, and in poly(methyl methacrylate) (PMMA). In PMMA films, the excitation spectrum is much the same as observed in fluid solution at room temperature. In Zeonex and the polythene melt, there was a slight increase in spectral fine-structure but the overall effect was not so significant. The stretched film appears to provide the requisite combination of immobilisation, single environment and polarity that results in the molecule being restored to its mirror-symmetry state. Comparable results were recorded for BOPHY under the same conditions.
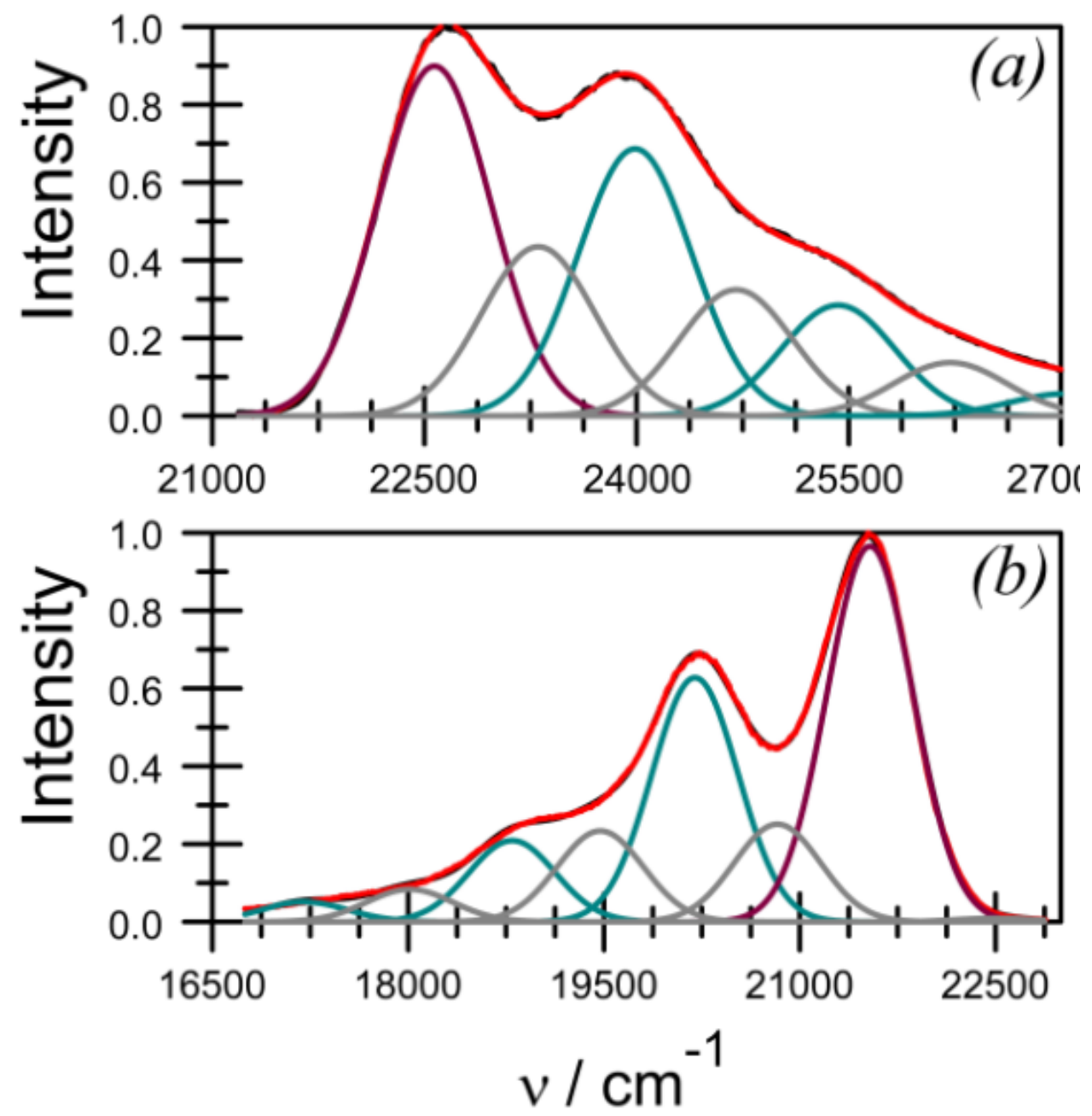

Figure 5. Illustration of the spectral deconstruction procedure used to extract information about the vibronic modes accompanying optical transitions for TM-BOPHY in the stretched film. The spectra were recorded at $130 \mathrm{~K}$ and refer to (a) reduced fluorescence and (b) reduced excitation profiles. The black curve is the experimental spectrum while the overlaid red curve is the re-constructed spectrum. The 0,0 transition appears as the plum curve while the low-frequency and medium-frequency modes are shown as grey and blue curves, respectively.

The stretched film provides a relatively simple medium with which to examine the effects of temperature on the emission and excitation spectra because it is unlikely that the polyethylene will undergo changes in polarizability during the temperature run. Consequently, fluorescence and excitation spectra were recorded for TM-BOPHY in a stretched film at temperatures from $80 \mathrm{~K}$ to $290 \mathrm{~K}$. The reduced spectra were subjected to analysis in terms of a vibrational progression on the basis of Gaussian band shapes. It was found necessary to include both low-frequency, around $600 \mathrm{~cm}^{-1}$, and medium-frequency, around $1,275 \mathrm{~cm}^{-1}$, vibronic modes to fully represent the reduced spectra (Figure 5). It was not possible, for example, to use only a medium-frequency vibration of around 1,500-1,600 
$\mathrm{cm}^{-1}$. This latter mode would correspond to stretching of the central N-N bond, which has been recognized by quantum chemical calculations as playing an important role in radiationless decay [9, 14]. Our analysis, carried out across the entire temperature range, requires the low-frequency mode as well as the accompanying medium-frequency vibration for both sets of spectra. It should be emphasized, however, that the spectral deconstruction procedure gives rise to averaged vibronic modes.

The derived spectroscopic records were analysed to give numerical values for the energy of the 0,0 transitions $\left(E_{00}\right)$, the low- $\left(h \omega_{L}\right)$ and medium-frequency $\left(h \omega_{M}\right)$ modes, and the respective Huang-Rhys $\left(S_{L}\right.$ and $\left.S_{M}\right)$ factors [20] as a function of temperature. The analysis also gives the full-width at halfmaximum (FWHM) for the resolved vibronic modes. Although there is overlap between $h \omega_{M}$ and the first overtone for $h \omega_{L}$, the latter is reasonably harmonic and readily isolated from the mediumfrequency value. Figure 6 illustrates how these various parameters are affected by temperature. For the reduced fluorescence spectra, $E_{00}$ remains at $20,415 \mathrm{~cm}^{-1}$ at all temperatures while the energies of the two vibrations are insensitive to changes in temperature. The band half-width increases steadily with increasing temperature, representing small changes in re-organisation energy [21], while the Huang-Rhys factors also show a shallow dependence on temperature [22]. Overall, the reduced fluorescence spectra do not change unduly with temperature and it appears that, at least in the stretched film, the ground-state geometry is not significantly affected by temperature. This situation is doubtless simplified by the absence of solvent effects.

The same analysis was applied to the reduced excitation spectrum and the main results are summarised by way of Figure 6 . Here, Eoo increases slightly with increasing temperature, indicating that the excited-singlet state becomes less planar as the temperature rises [23]. The combined effect on the respective $E_{00}$ energies is a rather gradual increase in the magnitude of the Stokes shift with rising temperature. Although the medium-frequency mode remains constant at ca. $1,330 \mathrm{~cm}^{-1}$, the energy of the corresponding low-frequency mode increases exponentially with temperature. This variation is quite significant, showing an increase from $530 \mathrm{~cm}^{-1}$ at $80 \mathrm{~K}$ to $680 \mathrm{~cm}^{-1}$ at $290 \mathrm{~K}$. There are also important variations for the band half-width and Huang-Rhys factor for the low-frequency mode that are more significant than found from the fluorescence spectra. The net result is that excitation causes the $S_{1}$ state to undergo important structural changes that combine to lose planarity. The geometry of the ground-state is less affected by temperature. The low-frequency vibronic mode represents a largescale structural distortion, such as ring inversion, while the medium-frequency vibronic mode is likely associated with $\mathrm{C}=\mathrm{C}, \mathrm{C}=\mathrm{N}$ and/or $\mathrm{N}=\mathrm{N}$ stretching vibrations [24]. It is this lowfrequency mode that shows the more significant temperature dependence. Before attempting to rationalise these findings, attention was turned to the corresponding studies made in solution. 


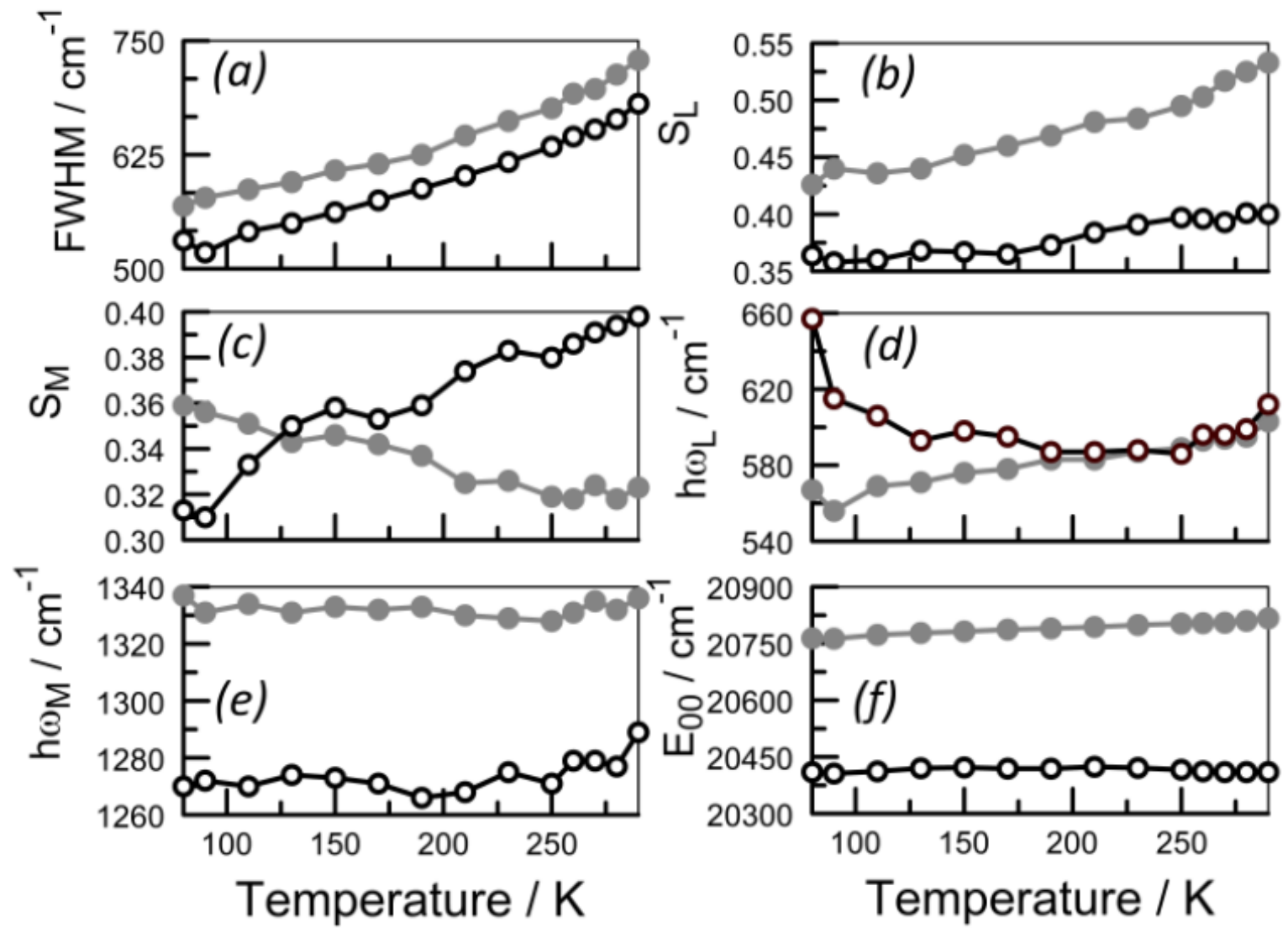

Figure 6. Effect of temperature on the various spectroscopic properties derived by the spectral deconstruction protocol as applied to TM-BOPHY in the stretched film. The parameters are (a) the fullwidth at half maximum, (b) the Huang-Rhys factor for the low-frequency mode, (c) the Huang-Rhys factor for the medium-frequency mode, (d) the low-frequency mode, (e) the medium-frequency mode and (f) the energy of the 0,0 transition. In each case, the open circles refer to the fluorescence spectrum and the grey spheres refer to the corresponding excitation spectrum.

\section{Temperature effects on the reduced fluorescence spectrum in MTHF}

The above spectral deconstruction analysis could be applied to fully reconstruct the reduced emission spectrum recorded for TM-BOPHY in MTHF solution across the temperature range from $80 \mathrm{~K}$ to $290 \mathrm{~K}$. However, it proved necessary to include a hot-fluorescence band at all temperatures to properly explain the high-energy edge of the spectrum. The significance of this hot emission increased slightly with temperature, with the peak maximum being in located at $500-700 \mathrm{~cm}^{-1}$ above the 0,0 band. At all temperatures, the residual spectrum could be well explained in terms of low- and mediumfrequency vibronic modes. It might be recalled that the solvent forms a rigid glass at temperatures below around $100 \mathrm{~K}$ and melts at $137 \mathrm{~K}$ [18]. Between these two limits, MTHF exists as an amorphous glassy matrix. Fluorescence spectra were recorded for each of the three phases and analysed as above. The main results are summarised by way of Figure 7 and presented in more detail in the Supporting Information. The same analysis was carried out for the reduced excitation spectra and the key findings are also shown as part of Figure 6. Closely comparable findings were recorded with BOPHY under the same conditions (see Figures S6 and S7). 

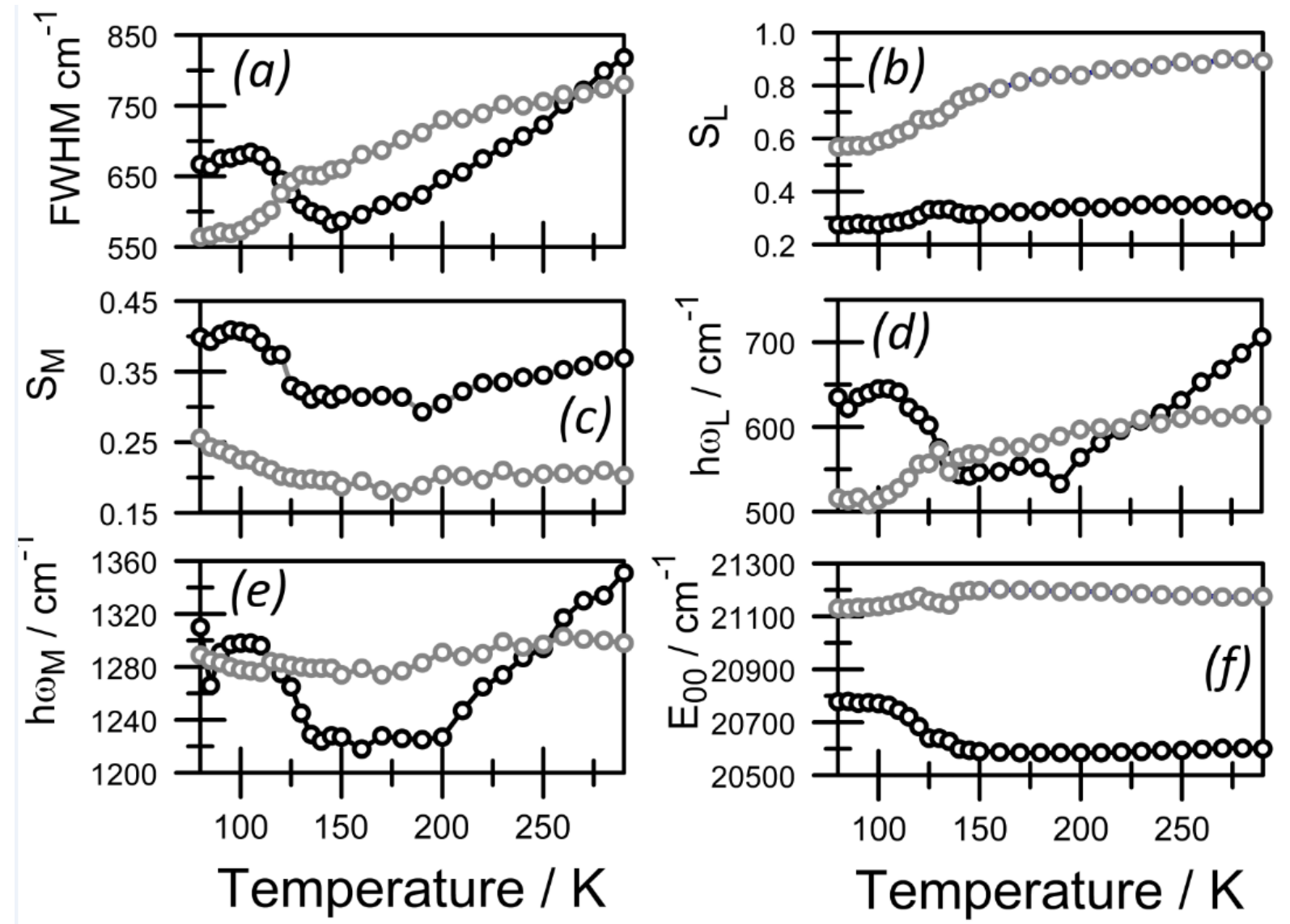

Figure 7. Effect of temperature on the various spectroscopic properties derived by the spectral deconstruction protocol as applied to TM-BOPHY in MTHF solution. The parameters are (a) the fullwidth at half maximum, (b) the Huang-Rhys factor for the low-frequency mode, (c) the Huang-Rhys factor for the medium-frequency mode, (d) the low-frequency mode, (e) the medium-frequency mode and (f) the energy of the 0,0 transition. In each case, the open circles refer to the fluorescence spectrum and the grey spheres refer to the corresponding excitation spectrum.

Unlike the case with the stretched film, temperature affects the polarizability of the solvent [25] and this effect is manifest in a certain degree of instability in the derived parameters near the glass transition temperature $\left(T_{G}=100 \mathrm{~K}\right)$ and at the freezing point $\left(T_{F}=137 \mathrm{~K}\right)$. The $E_{00}$ term, in particular, reflects the phase of the solvent and changes markedly as the amorphous glass melts. The net result is a substantial change in the magnitude of the Stokes shift on passing from glassy matrix to fluid solution. The full-width at half-maximum also tracks changes in the state of the solvent and increases steadily with increasing temperature in fluid solution. The Huang-Rhys factor for the mediumfrequency vibronic mode is relatively insensitive to temperature, although there is a slight correlation with the phase. That for the low-frequency mode exhibits a more pronounced temperature dependence, especially for the excitation spectra. The magnitude of both vibronic modes increases systematically with increasing temperature for the excitation spectrum but there is more instability in the derived values for the corresponding fluorescence spectra. In most cases, there is an important change in these vibrations as the glass melts. The most significant changes, therefore, are seen as the solvent enters or leaves the glassy matrix. It is this region that controls the mirror symmetry.

Essentially the same behaviour is observed for the parent compound, BOPHY, but the various spectroscopic parameters are somewhat more pronounced. For example, the band half-width together with both $S_{L}$ and $h \omega_{L}$ are significantly larger for BOPHY than for TM-BOPHY across the 
temperature range (see Supporting Information). The methyl groups lower Eoo for both excitation and fluorescence processes and decrease the Stokes shift by several hundred wavenumbers. As found for TM-BOPHY, the magnitude of the medium-frequency vibronic mode is relatively insensitive to temperature while $S_{M}$ tends to decrease with increasing temperature. Again, this latter effect is more exaggerated for BOPHY than for TM-BOPHY.

Overall, the temperature effects on the optical spectroscopic properties are fully consistent with the chromophore undergoing small structural fluctuations in fluid solution [22]. These changes are dampened out in the rigid glass and hampered in the amorphous phase but are switched on in fluid solution. The methyl substituents also help to dampen the accompanying vibrations. The Huang-Rhys factors report on the extent of structural disorder [26] and decrease with increasing planarity [27]. In more extended molecules, these factors can be used to decipher the effective conjugation length [28] but for smaller molecules, such as BOPHY, this is not really the case. With decreasing temperature, the shift towards planarity favours a larger delocalisation pathway for the electrons and affects the degree of electron-photon coupling. This is more pronounced for the low-frequency vibronic mode, where the coupling constant increases steadily with temperature. The latter situation arises when the energy barrier for the torsional mode is somewhat larger than the energy of the vibrational energy [29]. Indeed, analysis of the low-frequency mode in terms of Equation 1 allows estimation of the torsional barriers (E) and the Huang-Rhys factors at "low" temperature for the chromophore in the stretched film (Table 3; Figure 8). Here, $\mathrm{B}$ is an adjustable coefficient and $\mathrm{n}_{0}$ is the effective conjugation length for the molecule; the latter parameter should be close to zero for a small isolated molecule. The principle here is that $S_{L}$ is at a minimum for the completely planar geometry.

$$
\begin{gathered}
S_{L}=S_{L}^{0}+\frac{B}{\left(n_{0} e^{\frac{E}{R T}}\right)} \\
S_{L}=S_{L}^{0} e^{-\frac{E}{R T}}
\end{gathered}
$$

Table 3. Compilation of the structural parameters derived from Equations 1 or 2 as applied to the low-

\begin{tabular}{|c|c|c|c|c|}
\hline System & $S_{L}{ }^{0}$ & $\mathrm{E} / \mathrm{cm}^{-1}$ & $\mathbf{n}_{0}$ & B \\
\hline TM-BOPHY/SF/FL ${ }^{(b)}$ & 0.36 & 1,260 & 0.0001 & 0.040 \\
\hline TM-BOPHY/SF/EX ${ }^{(b)}$ & 0.44 & 1,350 & 0.0001 & 0.085 \\
\hline TM-BOPHY/SOL/FL (c) & 0.40 & 25 & NA & NA \\
\hline TM-BOPHY/SOL/EX ${ }^{(c)}$ & 1.07 & 35 & NA & NA \\
\hline BOPHY/SOL/FL ${ }^{(c)}$ & 0.60 & 60 & NA & NA \\
\hline $\mathrm{BOPHY} / \mathrm{SOL} / \mathrm{EX}^{(\mathrm{c})}$ & 1.03 & 70 & NA & NA \\
\hline
\end{tabular}
frequency vibronic mode. ${ }^{\text {a }}$

(a) $\mathrm{SF}=$ stretched film; FL = fluorescence; $E X=$ excitation. (b) Refers to Equation 1. (c) Refers to Equation 2. 

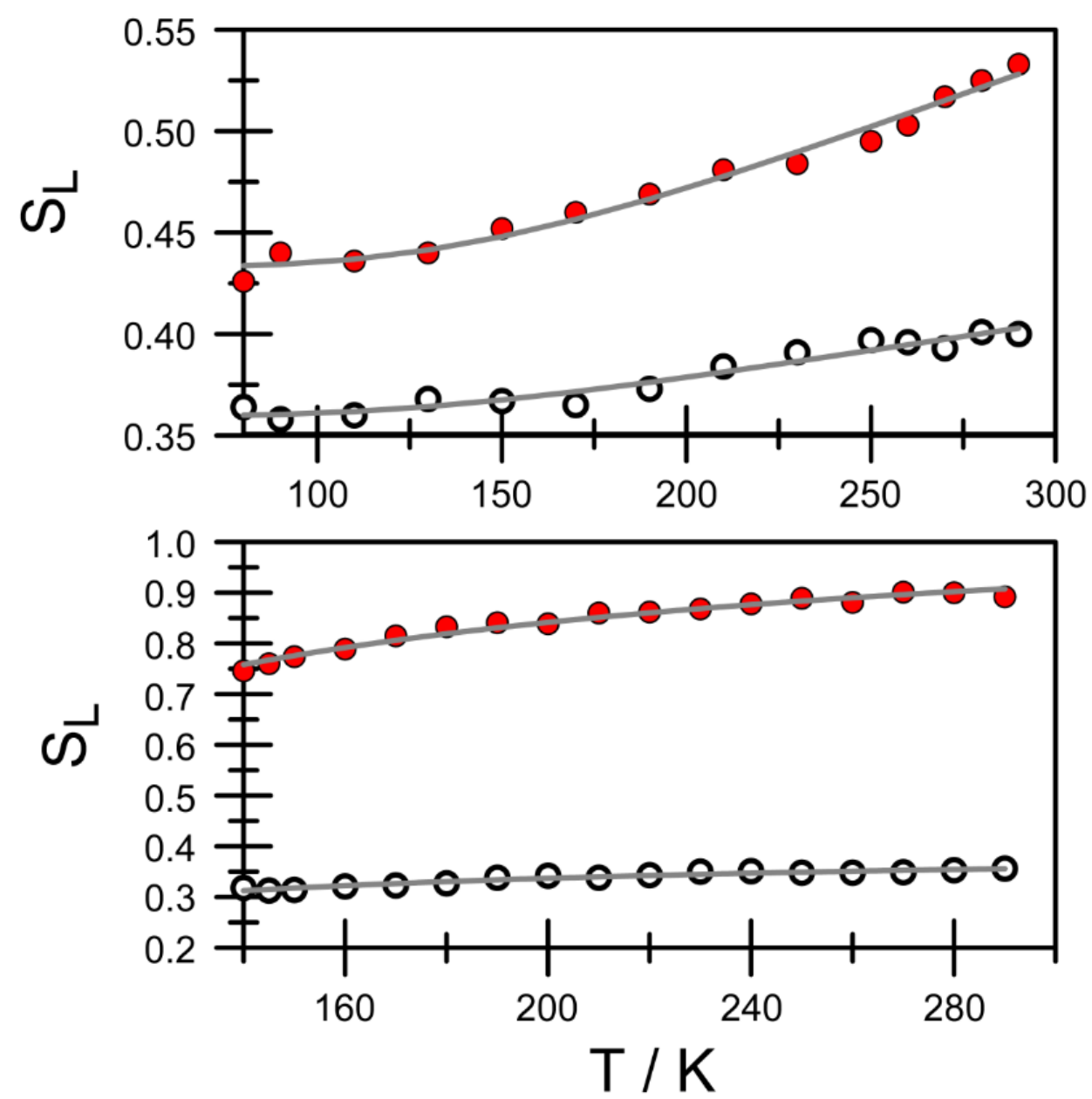

Figure 8. Effect of temperature on the magnitude of the Huang-Rhys factor for the low-frequency vibronic mode. The upper panel refers to TM-BOPHY in a stretched film, where the fit to the experimental data is in accordance with Equation 1. The lower panel refers to TM-BOPHY in liquid MTHF, with the fit corresponding to Equation 2. In all cases, the open circles refer to fluorescence and the red points refer to excitation data.

In the stretched film, Equation 1 can be used to correlate $S_{L}$ with absolute temperature for both emission and excitation spectra (Table 3 ) but this is not the case for fluid solution. Indeed, fitting the experimental data to Equation 1 leads to unrealistic fitting parameters, most notably negative $S_{L}$ values at the limiting lower temperature. However, $\mathrm{S}_{\mathrm{L}}$ for both excitation and fluorescence data can be well explained in terms of Equation 2 where $E$ represents a low-energy barrier. The derived parameters for TM-BOPHY and BOPHY are given in Table 3 and examples of the fits are given in Figure 8. In all cases, the limiting $S_{L}{ }^{0}$ value lies close to unity and the barriers are very small. The energy barriers are extremely small. Comparing data derived for TM-BOPHY in a stretched film and fluid solution shows that the significant change is associated with the excitation spectrum, where the limiting $S_{L}$ is rather high. A comparable value is found for BOPHY. This indicates that excitation leads to a distorted (i.e., non-planar) geometry. The barriers are introduced by the solvent and, although small, help to control the band-shape.

The medium-frequency mode is much less sensitive to temperature. In fact, $\mathrm{S}_{M}$ might decrease slightly with increasing temperature in most cases while the magnitude of $h \omega_{M}$ shows a slight tendency to 
increase as the temperature rises in solution. Such behaviour has been noted previously for conjugated polymers $[28,30]$. The energy of the vibration depends on the associated force constants and reduced masses. In general increasing molecular planarity tends to lower the frequency of the accompanying vibrations, this is certainly the case for polyacetylenes [31] and carotenoids [32], and this crude generalisation appears to hold also for BOPHY in fluid solution.

\section{NMR Spectroscopy}

To search for the possible involvement of different molecular conformations, a series of temperature dependent NMR spectra were recorded for TM-BOPHY in $\mathrm{d}_{8}$-toluene (see Supporting Information). Proton spectra were run over the temperature range 193-353K; at all temperatures, there was just one set of peaks. The methyl protons at $1.65 \mathrm{ppm}$ and the Ar-H at $5.63 \mathrm{ppm}$ shift by 0.39 and 0.33 $\mathrm{ppm}$, respectively, over the full temperature range. These shift changes may be due to changes in the extent of association between the solvate and the aromatic solvent [33] and/or to differing proportions of rapidly interconverting conformers. There is no evidence of non-interconverting conformers. It is reasonable to suppose that the occurrence of non-interconverting conformers at low temperature would be more evident from NMR spectra of the ${ }^{19} \mathrm{~F}$ nucleus. Unfortunately, due to the increased broadening brought about by the ${ }^{11} \mathrm{~B}$ quadrupole, the effective resolution of the ${ }^{19} \mathrm{~F}$ spectrum at $193 \mathrm{~K}$ is insufficient to observe any such behaviour.

To search for the possible involvement of different molecular conformations, a series of temperaturedependent NMR spectra were recorded for TM-BOPHY in $d_{8}$-toluene (see Supporting Information). At $500 \mathrm{MHz}$, the proton NMR spectra do not change over a temperature range from 193 to $353 \mathrm{~K}$. There is no evidence for more than one species being present over this temperature range. It is reasonable to suppose that the occurrence of non-interconverting conformers at low temperature would be more evident from NMR spectra of the ${ }^{19} \mathrm{~F}$ nucleus. Relevant spectra were recorded at $298 \mathrm{~K}$ and $193 \mathrm{~K}$, and it appears that at lower temperatures there is a progressive loss of coupling from the ${ }^{11} \mathrm{~B}$ nucleus to the ${ }^{19} \mathrm{~F}$ nucleus as a result of the faster relaxation of the ${ }^{11} \mathrm{~B}$ nucleus. Under these conditions, the broadness arising from the nearby ${ }^{11} \mathrm{~B}$ nucleus limits the experimental resolution but it does not appear that multiple conformers are in equilibrium in solution. On this basis, the observed changes in the optical properties reflect fluctuations in the molecular topology of a single entity.

This finding, together with the realisation that the changes in band shapes for the optical spectra can be accommodated in terms of modification of the vibronic envelope, indicates that the absorption (and excitation) band seen at around $450 \mathrm{~nm}$ is due to a single transition. Ultrafast transient absorption spectroscopy applied to BOPHY and TMBOPHY in fluid solution had already reached the same conclusion [11]. On the basis of TD-DFT calculations, Ziegler et al. [5] concluded that the absorption spectra for BOPHY and TM-BOPHY were best explained in terms of a single electronic transition with a vibronic progression. Furthermore, they argue that the absorption band centred at around $400 \mathrm{~nm}$ is primarily due to HOMO-LUMO excitation. Our NMR studies rule out the likelihood of multiple conformers with slightly different HOMO-LUMO energy gaps.

\section{Conclusion}

The coupling of optical properties with light-induced geometrical change is of especial importance for conjugated polymers where it has been recognised that low-frequency vibronic modes can make significant contributions to the spectral band-shape. Our Frank-Condon analysis requires both medium- and low-frequency vibrations in order to adequately reproduce the observed temperature dependence for fluorescence and excitation spectra recorded for BOPHY and TM-BOPHY. For the lowfrequency mode, the electron-photon coupling increases monotonically with temperature whereas 
the medium-frequency mode is strongly affected by changes in the solvent properties. It is difficult to disentangle solvent and structural effects for the solution-phase studies but it is clear that the geometry change that accompanies melting of the solvent causes the loss of mirror symmetry between excitation and fluorescence spectra. There are no such structural changes for TM-BOPHY incorporated into the stretched film and, because of the absence of solvent polarizability effects, the vibrational data are more easily related to structural changes. In fact, the derived parameters for the stretched film evolve gently with temperature and, in general, will combine to broaden the spectra. Thus, there is no serious spectral change as the temperature increases.

On moving to solution phase, there is a general increase in both band half-width and $\mathrm{E}_{00}$ values with temperature. These effects are directly related to solute-solvent interactions. The presence of the solvent has only a slight effect on the magnitude of the vibronic modes and on $\mathrm{S}_{\mathrm{M}}$. There is a larger change between $S_{L}$ values found in the stretched film and in solution for the excitation spectra. Furthermore, these latter changes track the phase change of the solvent and it is this solvent-induced modulation of $S_{L}$ that is primarily responsible for the loss of mirror symmetry. The corresponding fluorescence spectra exhibit a much more restrained temperature dependence for $\mathrm{S}_{\mathrm{L}}$, thereby indicating that the dominant structural change accompanies excitation in fluid solution. This is so for both BOPHY and TM-BOPHY.

This new class of pyrrole- $\mathrm{BF}_{2}$ dyes is promising in that it affords synthetic opportunities not easily available to BODIPY. Substitutions can be made at opposite corners of the core structure, allowing for long, linear molecules. High, although non-unity, fluorescence quantum yields are found, while the absorption spectrum is rather broad. This latter feature is a desirable property for molecules that act as quantum yield standards and for light-harvesting purposes. The BOPHY chromophore is fairly resistant to photodegradation in both fluid solution and plastic films, although auto-catalysis becomes important at long illumination periods [33], and has been reported to facilitate long-range electronic coupling between distal terminals [34,7b]. We might anticipate a growing usage of the BOPHY unit in multi-component molecular systems aimed at advanced opto-electronic devices.

\section{Acknowledgement}

We thank Newcastle University for financial support of this work. The EPSRC is gratefully acknowledged for the award of an Industrial CASE Award to OJW and AAA thanks Shaqra University of Saudi Arabia for a research scholarship.

\section{Supporting Information}

Supporting information gives experimental details, compound characterisation, examples of NMR spectra recorded at different temperatures, examples of absorption and fluorescence spectra recorded in fluid solution, experimental data underlying Figure 6 and also for the corresponding studies made with BOPHY.

\section{References}

[1] A. Loudet, K. Burgess, BODIPY dyes and their Derivatives: Synthesis and Spectroscopic properties, Chem. Rev. 107 (2007) 4891-4932.

[2] G. Ulrich, R. Ziessel, A. Harriman, The chemistry of bodipy dyes: Versatility unsurpassed, Angew. Chem., Int. Ed. 47 (2008) 1184-1201.

[3] N. Boens, V. Leen, W. Dehaen, Fluorescent Indicators based on BODIPY, Chem. Soc. Rev. 41 (2012) 1130-1172. 
[4] R. Ziessel, G. Ulrich, A. Harriman, The chemistry of Bodipy: A new El Dorado for fluorescence tools, New J. Chem. 31 (2007) 496-501.

[5] I.S. Tamgho, A. Hasheminasab, J.T. Engle, V.N. Nemykin, C.J. Ziegler, A new highly fluorescent and symmetric pyrrole-BF2 chromophore: BOPHY, J. Am. Chem. Soc. 136 (2014) 5623-5626.

[6] Q. Hualme, A. Mirloup, P. Retailleau, R. Ziessel, Synthesis of highly functionalized BOPHY chromophores displaying largeStokes shifts, Org. Lett. 17 (2015) 2246-2249.

[7] a) X.D. Jiang, Y.J. Su, S. Yue, C. Li, H. F. Yu, H. Zhang, C.L. Sun, L.J. Xiao, Synthesis of mono-(pdimethylamino)styryl-containing BOPHY dye as a turn-on pH sensor, RSC Adv. 5 (2015) 16735-16739.

b) O.J. Woodford, P. Stachelek, R. Ziessel, N. Algoazy, J.G. Knight, A. Harriman, End-to-end communication in a linear supermolecule with a BOPHY centre and $\mathrm{N}, \mathrm{N}$-dimethylanilino terminals, New J. Chem. 42 (2018) 4835-4842.

[8] a) J. Wang, Q.H. Wu, C.J. Yu, Y. Wei, X.L. Mu, E.H. Hao, L.J. Jiao, Aromatic ring fused BOPHYs as stable red fluorescent dyes, J. Org. Chem. 81 (2016) 11316-11323.

b) L. Zhou, D.F. Xu, H.Z. gao, C. Zhang, F.F. Ni, W.Q. Zhao, D.D. Cheng, X.L. Liu, A.X. Han, betaFuran-fused bis(difluoroboron)-1,2-bis(1H-pyrrol-2-yl)methylene)hydrazine fluorescent dyes in visible deep-red region, J. Org. Chem. 81 (2016) 7439-7447.

c) Zatsikha, D.B. Nemez, R.L. Davis, S. Singh, D.E. Herbert, A.J. King, C.J. Ziegler, V.N. Nemykin, Testing the limits of the BOPHY platform: Preparation, characterization, and theoretical modelling of BOPHYs and organometallic BOPHYs with electron-withdrawing groups at beta-pyrrole and bridging positions, Chem. Eur. J. 23 (2017) 14786-14796.

d) S. Boodts, J. Hofkens, W. Dehaen, Unprecedented alpha-substituted BOPHY dyes via a key 3,8dichloroBOPHY intermediate, Dyes Pigm. 142 (2017) 249-254.

[9] M. Ponce-Vargas, C. Azarias, Jacquemin, B. Le Guennic, Combined TD-DFT-SOS-CIS(D) study of BOPHY derivatives with potential application in biosensing, J. Phys. Chem. B 121 (2017) 10850-10858.

[10] C.S. Zhang, J.Z. Zhao, Triplet excited state of diiodoBOPHY derivatives: Preparation, study of photophysical properties and application in triplet-triplet annihilation upconversion, J. Mater. Chem. C 4 (2016) 1623-1632.

[11] L. Wang, I.S. Tamgho, L.A. Crandall, J.J. Rack, C.J. Ziegler, Ultrafast dynamics of a new class of highly fluorescent boron difluoride dyes, Phys. Chem. Chem. Phys. 17 (2015) 2349-2351.

[12] G.J. Hedley, A. Ruseckas, A. Harriman, I.D.W. Samuel, Conformational effects on the dynamics of internal conversion in boron dipyrromethene dyes in solution, Angew. Chem., Int. Ed. 50 (2011) 66346637.

[13] P. Toele, H. Zhang, C. trieflinger, J. Daub, M. Glasbeek, Femtosecond fluorescence upconversion study of a boron dipyrromethene dye in solution, Chem. Phys. Lett. 368 (2003) 66-75.

[14] R. Zalesny, N.A. Murugan, G. Tian, M. Medved, H. Agren, First-principles simulations of one- and two-photon absorption band shapes of the bis(BF2) core complex, J. Phys. Chem. B 120 (2016) 23232332.

[15] S.J. Strickler, R.A. Berg, Relationship between absorption intensity and fluorescence lifetime of molecules, J. Chem. Phys. 37 (1962) 814-822.

[16] R. Bosque, J. Sales, Polarizabilities of solvents from chemical composition, J. Chem. Inf. Comput. Sci. 42 (2002) 1154-1163.

[17] J. Häslmayr, T. Renger, Qualitative change of character of dispersive interaction with intermolecular distance, J. Chem. Phys. 139 (2013) 044103. 
[18] T. Rong-Ri, S. Xin, H. Lin, Z. Feng-Shou, Liquid-to-glass transition of tetrahydrofuran and 2methyltetrahydrofuran, Chin. Phys. B 21 (2012) 086402.

[19] M. Levitus, M.A. Garcia-Garibay, Polarized electronic spectroscopy and photophysical properties of 9,10-bis(phenyl)anthracene, J. Phys. Chem. A 104 (2000) 8632-8637.

[20] a) J. Gierschner, H.-G. Mack, L. Lúer, D. Oelkrug, Fluorescence and absorption spectra of oligophenylenevinylenes: Vibronic coupling, band shapes and solvatochromism, J. Chem. Phys. 116 (2002) 8596-8609.

b) F.A.C. Oliveira, L.A. Cury, A. Righi, R.L. Moreira, P.S.S. Guimarães, F.M. Matinaga, M.A. Pimenta, R.A. Nogueira, Temperature effects on the vibronic spectra of BEH-PPV conjugated polymer films, J. Chem. Phys. 119 (2003) 9777-9782.

c) A. Marletta, F.E.G. Guimarães, R.M. Faria, Line shape of emission spectra of the luminescent polymer ploy(p-phenylene vinylene), Braz. J. Phys. 32 (2002) 570-574.

d) C.A.M. Borges, A. Marletta, R.M. Faria, F.E.G. Guimarães, Vibrational structure of organic semiconductors: The role of conjugation length, Braz. J. Phys. 34 (2004) 590-592.

[21] G. Zucchelli, F.M. Garlaschi, R.C. Jennings, Thermal broadening analysis of the light harvesting complex II absorption spectrum, Biochem. 35 (1996) 16247-16254.

[22] R. Al-aqar, A.C. Benniston, A. Harriman, T. Perks, Structural dynamics and barrier crossing observed for a fluorescent O-doped polycyclic aromatic hydrocarbon, ChemPhotoChem 1 (2017) 198205.

[23] K. Roy, S. Kayal, F. Ariese, A. Beeby, Mode specific excited state dynamics study of bis(phenylethynyl)benzene from ultrafast Raman loss spectroscopy, J. Chem. Phys. 146 (2017) 064303.

[24] F. Zimmermann, Th. Lippert, Ch. Beyer, J. Stebani, O. Nuyken, A. Wokaun, N=N Vibrational frequencies and fragmentation patterns of substituted 1-aryl-3,3-dialkyl-triazenes: Comparison with other high-nitrogen compounds, Appl. Spectrosc. 47 (1993) 986-993.

[25] P.O. Andersson, S.M. Bachilo, R.L. Chen, T. Gillbro, Solvent and temperature effects on dual fluorescence in a series of carotenes - Energy gap dependence of the internal-conversion rate, J. Phys. Chem. 99 (1995) 16199-16209.

[26] J. Schutze, B. Bruggemann, T. Renger, V. May, Theory of linear absorption spectra of biological and non-biological chromophore complexes, Chem. Phys. 275 (2002) 333-354.

[27] N.H. Damrauer, T.R. Boussie, M. Devenney, J.K. McCusker, Effects of intraligand electron delocalization, steric tuning, and excited-state vibronic coupling on the photophysics of arylsubstituted bipyridyl complexes of Ru(II), J. Am. Chem. Soc. 119 (1997) 8257-8268.

[28] L. O'Neill, H.J. Byrne, Structure-property relationships for electron-vibrational coupling in conjugated organic oligomeric systems, J. Phys. Chem. B 109 (2005) 12685-12690.

[29] F.C. Spanno, L. Silvestri, , J. Chem. Phys. 132 (2010) 094704.

[30] T.W. Hagler, K. Pakbaz, K.F. Voss, A.J. Heeger, Enhanced order and electronic delocalization in conjugated polymers oriented by gel processing in polyethylene, Phys. Rev. B 44 (1991) 8652-8666.

[31] a) I. Harada, M. Tasumi, H. Shirakawa, S. Ikeda, Raman spectra of polyacetylene and highly conducting iodine-doped polyacetylene, Chem. Lett. 12 (1978) 1411-1414.

b) I. Harada, Y. Furakawa, M. Tasumi, H. Shirakawa, S. Ikeda, Spectroscopic studies of polyacetylene and beta-carotene, J. Chem. Phys. 73 (1980) 4746-4757.

[32] H. Kuzmany, Resonance Raman scattering from neutral and doped polyacetylene, Phys. Status Solidi B 97 (1980) 521-531. 
[33] O.J. Woodford, A. Harriman, W. McFarlane, C. Wills, Dramatic effect of solvent on the rate of photobleaching of organic pyrrole-BF2 (BOPHY) dyes, ChemPhotoChem 1 (2017) 317-325.

[34] H.M. Rhoda, K. Chanawanno, A. J. King, Y. V. Zatsikha, C. J. Ziegler, V.N. Nemykin, Unusually strong long-distance metal-metal coupling in bis(ferrocene)-containing BOPHY: An introduction to organometallic BOPHYs, Chem. Eur. J. 21 (2015) 18043-18046.

\section{Table of Contents Figure}

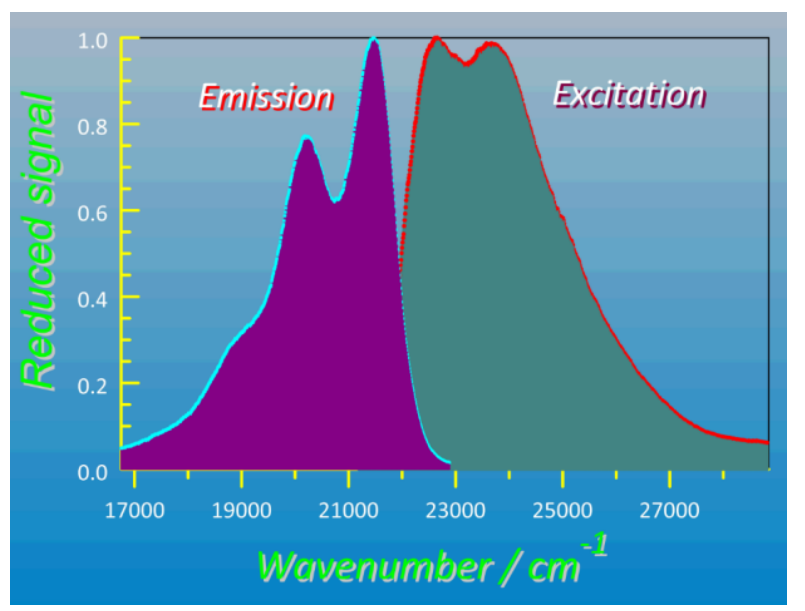

\section{Highlights for Ms. Ref. No.: SAA-D-18-01744}

Excitation and fluorescence spectra have been recorded for the target chromophores at temperatures ranging from $80 \mathrm{~K}$ to slightly above room temperature.

Optical spectra have also been recorded with the compound embedded in a stretched polythene film.

Spectra have been subjected to a detailed curve-fitting analysis to expose the Huang-Rhys factors, Franck-Condon vibronic modes and 0,0 transition energies as a function of temperature.

A key feature of this analysis relates to the involvement of a low-frequency vibration that is instrumental in causing a loss of mirror symmetry under ambient conditions.

Mirror symmetry is restored in solid media where local structural perturbations are hindered. 